\title{
Clareamento dental simplificando o protocolo clínico: relato de caso clínico
}

\author{
Tooth whitening simplifying the clinical protocol: clinical case report \\ Blanqueamiento dental simplificando el protocolo clínico: reporte de caso clínico
}

Recebido: 23/11/2021 | Revisado: 30/11/2021 | Aceito: 01/12/2021 | Publicado: 01/12/2021

\author{
Adiel de Oliveira Castelo \\ ORCID: https://orcid.org/0000-0002-1416-9312 \\ Centro Universitário Fametro, Brasil \\ E-mail: adiel.castelo2@gmail.com \\ Andrew Henrique Dias de Andrade \\ ORCID: https://orcid.org/0000-0002-0872-2783 \\ Centro Universitário Fametro, Brasil \\ E-mail: andrew.andrade1005@gmail.com \\ Mateus da Silva Gomes \\ ORCID: https://orcid.org/0000-0002-5283-1602 \\ Centro Universitário Fametro, Brasil \\ E-mail: mateusdasilvagomes2526@gmail.com \\ Gabriela de Figueiredo Meira \\ ORCID: https://orcid.org/0000-0002-8285-8769 \\ Universidades Federal de Santa Maria, Brasil \\ E-mail: gabrielameira1@hotmail.com \\ Thiago Mendes de Lima \\ ORCID: https://orcid.org/0000-0001-6524-3271 \\ Centro Universitário Fametro, Brasil \\ E-mail: thiago.lima@fametro.edu.br
}

\begin{abstract}
Resumo
Objetivo: Este estudo tem como objetivo simplificar o protocolo clínico do clareamento dental. Metodologia: Trata-se de um relato de caso clínico de cunho analítico descritivo (Pereira et al., 2018) Relato de caso clínico: Paciente MSG, sexo masculino, procurou a clínica de odontologia da Fametro buscando tratamento odontológico, onde através da anamnese identificou-se um desconforto com os dentes escurecidos, sendo possível diagnosticar o paciente e apresentar agumas opções de clareamento dental, onde o mesmo optou por clareamento de consultório. No exame bucal foi identificado restauração de resinas composta nos elementos 12 e 22. Assim sendo, na primeira sessão foi realizada a profilaxia e o registro de cor da escala Vita pan (Classical B1 - D4) com score A3, bem com a utilização do afastador labial e barreira gengival para proteção dos tecidos moles. Utilizou-se ainda o espelho clínico para verificar a existência de tecido descoberto e aplicado o dessensibilizante antes do gel clareador. Foi utilizado o agente clareador peróxido de hidrogênio a 35\% (Whitness HP Automixx / FGM) e após a ação do produto foi removido com cânula de sucção, lavado com água em abundância e removido a barreira gengival. Considerações finais: Desta forma os resultados encontados foram satisfatórios proporcionando um clareamento dental de A3 para B1, possibilitando um clareamento dental com um procedimento simples e pincipalmente com um tempo de intervalo reduzido de sete dias para três dias, proporcionando um resultado em menos tempo, mas com eficácia comprovada.
\end{abstract}

Palavras-chave: Clareamento dental; Estética dentária; Consultório odontológico.

\begin{abstract}
Objective: This study aims to simplify the clinical protocol of tooth whitening. Methodology: This is a clinical case report of descriptive analytical nature (Pereira et al., 2018) Clinical case report: MSG patient, male, sought the Fametro dental clinic seeking dental treatment, where he identified through the anamnesis - there is a discomfort with darkened teeth, making it possible to diagnose the patient and present some options for tooth whitening, where he opted for in-office whitening. In the oral examination, restoration of composite resins in elements 12 and 22 was identified. Therefore, in the first session, the prophylaxis and color registration of the Vita pan scale (Classical B1 D4) with a score of A3 was performed, as well as the use of a retractor lip and gingival barrier for soft tissue protection. The clinical mirror was also used to verify the existence of uncovered tissue and the desensitizer was applied before the whitening gel. A 35\% hydrogen peroxide bleaching agent (Whitness HP Automixx / FGM) was used and, after the action, the product was removed with a suction cannula, washed with plenty of water and the gingival barrier was removed. Final considerations: Thus, the results found were satisfactory, providing a tooth whitening from A3 to B1, enabling a tooth whitening with a simple procedure and mainly with a reduced interval time from seven days to three days, providing a result in less time, but with proven effectiveness.
\end{abstract}

Keywords: Tooth whitening; Dental aesthetics; Odontological office. 


\begin{abstract}
Resumen
Objetivo: Este estudio tiene como objetivo simplificar el protocolo clínico de blanqueamiento dental. Metodología: Se trata de un reporte de caso clínico de naturaleza analítica descriptiva (Pereira et al., 2018) Reporte de caso clínico: Paciente con MSG, de sexo masculino, acudió a la clínica dental Fametro en busca de tratamiento odontológico, donde identificó a través de la anamnesis - existe una incomodidad con dientes oscurecidos, lo que permitió diagnosticar al paciente y presentar algunas opciones de blanqueamiento dental, donde se optó por el blanqueamiento en consultorio. En el examen oral se identificó restauración de resinas compuestas en los elementos 12 y 22 . Por lo tanto, en la primera sesión, también se realizó la profilaxis y registro de color de la escala Vita pan (Clásica B1 - D4) con una puntuación de A3. como el uso de un retractor de labios y una barrera gingival para la protección de los tejidos blandos. También se utilizó el espejo clínico para verificar la existencia de tejido descubierto y se aplicó el desensibilizante antes del gel blanqueador. Se utilizó un agente blanqueador de peróxido de hidrógeno al 35\% (Whitness HP Automixx / FGM) y, después de la acción, se eliminó el producto con una cánula de succión, se lavó con abundante agua y se eliminó la barrera gingival. Consideraciones finales: Así, los resultados encontrados fueron satisfactorios, proporcionando un blanqueamiento dental de A3 a B1, posibilitando un blanqueamiento dental con un procedimiento sencillo y principalmente con un intervalo de tiempo reducido de siete días a tres días, proporcionando un resultado en menos tiempo, pero con eficacia probada.
\end{abstract}

Palabras clave: Blanqueamiento dental; Estética dental; Consultorio odontológico.

\title{
1. Introdução
}

A qualidade de vida das pessoas é influenciada pela saúde e a integridade bucal, onde existe a necessidade de cuidados em todas as faixas etária. Com o aumento da expectativa de vida e a busca por padrão de beleza, as pessoas passaram a almejar novas alternativas de reabilitação de um sorriso perfeito com segurança e bem estar (Poluha et. al., 2016). Dentes mais claros são cada vez mais almejados, devido a isso, odontologia foi criando novos métodos, novos procedimentos, novos materiais para se obter esse sorriso mais claro (Souza et al., 2020).

O clareamento dental ganhou uma grande aceitação por ser considerado um procedimento bastante simples, seguro, eficiente e bastante conservador para os dentes que apresentam alteração de cor. Porém, um dos efeitos adversos mais comuns do clareamento dental é a sensibilidade dentária, a qual está relacionado aos radicais livres gerados pela degradação dos peróxidos, que induzem uma resposta inflamatória pulpar; consiste em uma reação resultante da ação de mediadores inflamatórios associada a resposta neural dos odontoblastos, levando à ocorrência de dor (Rezende, et al., 2019; CaviedesBucheli et al., 2008; Markowitz et al., 2010; Kielbassa et al., 2015). Trata-se de uma sintomatologia específica, que se apresenta logo nos períodos iniciais do clareamento, de forma transitória, e está relacionada à sensação de dor espontânea, rápida e aguda, mesmo na ausência de dentina exposta (Kielbassa et al., 2015). Uma variedade de fatores relacionados à etiologia, fatores de risco e preditores são apresentadas a essa sintomatologia (Kielbassa et al., 2015), destacando-se o tempo de aplicação, concentração do gel e resposta individual do paciente, a qual é relatada por até dois terços dos pacientes que realizam o clareamento dental, com intensidade de leve a intolerável (Costa et al., 2010; Basting et al., 2012; Lima et al., 2019).

Atualmente existem dois métodos para a execução do clareamento de dentes vitais, sendo a técnica caseira, que utiliza o peróxido de carbamida em concentração de 10 a $20 \%$ em moldeiras e a técnica de consultório com peróxido de carbamida 35 a $37 \%$ ou peróxido de hidrogênio 30 a $35 \%$ as mais comumente empregadas pelos profissionais. Havendo a possibilidade de utilizar combinação das duas técnicas, como terceira opção sempre visando obter a harmonia estética que corresponda com as expectativas do paciente (Matinho e. al., 2019). Ambos os métodos pode ser indicado pelo profissional em odontologia, contudo devem ser supervisionado e colocando os limites ao paciente, esclarecendo todos os efeitos colaterais que podem se manisfestar no decorrer do tratamento tendo em vista a peculiaridade de cada indivíduo (Nascimento, Aracuri et al., 2018).

Durante o clareamento dental é importante conhecer os agentes clareadores, realizar uma excelente anamnese, diagnosticar o fator etilógico da alteração de cor assim como a analisar as particularidades de cada paciente tais como: os casos de pigmentação, idade, estado de vitalidade dos dentes a serem clareados. Esta análise pode facilitar a seleção correta da 
técnica clareadora, contudo é imprescindível que o profissional tenha um olhar clínico bastante específico, deixando os pacientes cientes que o resultado final do tratamento não é 100\% previsível (Neto et al., 2019). As alterações de cor podem ser causadas, basicamente, por dois fatores: extrínsecos, ocasionados pelo consumo excessivo de tabaco, alimentos e bebidas com corantes, como chá e café; e intrínsecos congênitos (dentinogênese imperfeita e a fluorose) ou adquiridos provenientes do uso indevido das tetraciclinas e do flúor durante a fase de formação dental, do envelhecimento fisiológico, da decomposição de sangue intracoronário, provocados por trauma acidental e falhas no tratamento endodôntico (Barbosa et al., 2020; Vieira et al., 2018; Neto et al., 2019).

Os mecanismos de ação dos agentes clareadores não são completamente compreendidos, mas basicamente, envolvem uma reação de oxidação, na qual as moléculas de peróxido de hidrogênio se quebram, liberando oxigênio e radicais livres de peridroxil, promovendo a quebra das macromoléculas pigmentadas em moléculas menores, promovendo o clareamento dos dentes (Franci et al., 2015)

Uma sociedade dinâmica, moderna e que supervaloriza a apresentação pessoal exige procedimentos com resultados mais rápidos. Neste contexto, este relato científico tem como objetivo principal apresentar um caso clínico sobre clareamento dental em consultório, com modificação do protocolo clínico da técnica de clareamento, com espaço entre as sessões reduzidos, levando a obteção dos resultados mais rápida e realizada de forma segura.

\section{Metodologia}

Partindo da permissão da paciente por meio do Termo de Consentimento Livre e Esclarecido (TCLE) em partilhar sua imagem para devido fim, o presente estudo trata de um relato de caso clínico de cunho analítico descritivo (Pereira et al., 2018), realizado na clínica odontológica do CEUNI Fametro, na disciplina de estágio supervisionado em Clínica Integrada II, do curso de graduação do Centro Universitário Fametro.

\section{Resultados e Discussão}

Paciente MSG ,23 anos, sexo masculino compareceu a Clínica Odontológica da Fametro para atendimento odontológico. Na anamnese relatou desconforto com a cor dos dentes escurecida. Relatou consumo de alimentos com corante alimentícios e dentre eles o consumo de café, refrigerante, sucos industrializados e entre outros todos os dias. Foram dadas opções para melhorar a estética do sorriso: clareamento caseiro, clareamento de consultório ou facetas de resinas compostas. O paciente optou pelo clareamento de consultório.

$\mathrm{Na}$ anamnese e exame clínico bucal dos elementos, foram necessárias radiografias periapicais para investigar a saúde pulpar e periodontal de alguns dos elementos. Foram encontradas restaurações de resina composta nos elementos 12 e 22 nas faces mediais e distais de ambos os elementos dentários citados.

Na primeira sessão foi realizada a profilaxia com pedra pomes e água. Seguido de registro de cor usando a escala de cor Vita Pan (Classical B1 - D4) com score A3 e registro fotográfico. (Figura 1). 
Figura 1: A) $1^{\text {a }}$ sessão antes; B) $1^{\text {a }}$ sessão depois do tratamento.

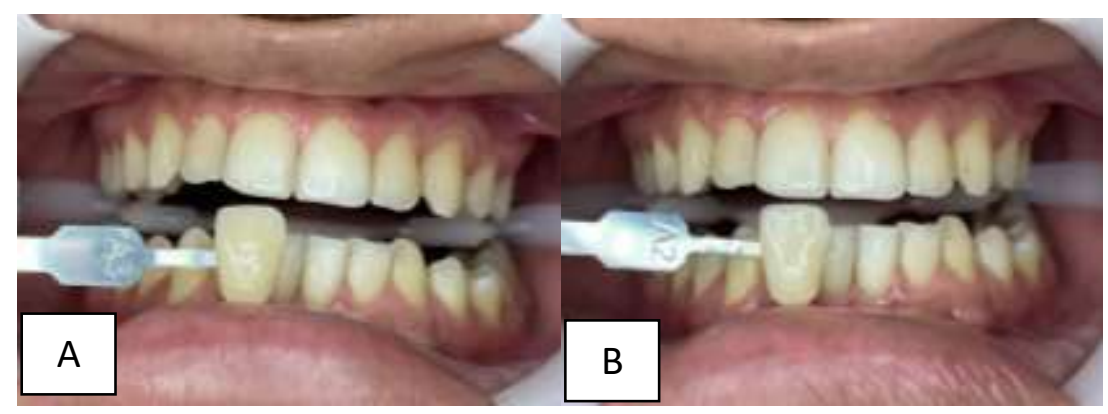

Fonte: Autores.

Como meio de proteção de tecidos moles evitando o contato do gel clareador com a mucosa foi utilizado afastador labial (ARC FLEX/ FGM) e em seguida barreira gengival fotopolimerizavél (TOP DAM/ FGM) para a proteção dos tecidos gengivais e cobrindo a gengiva com uma camada de 3 a $5 \mathrm{~mm}$ e $1 \mathrm{~mm}$ de espessura. Utilizou-se espelho clínico olhando de incisal para cervical para verificar se existe tecido gengival descoberto, caso seja identificado foi realizado ajuste (Figura 2). Antes da aplicação do gel foi aplicado o dessensibilizante (POTENZA ESENTE/ POTENZA) a 2\% por 10min. O agente clareador usado para o clareamento foi o peróxido de hidrogênio a 35\% (Whitness HP Automixx / FGM) aplicado apenas uma vez a cada sessão por tempo de 50 minutos. O produto foi aplicado de segundo pré-molar do lado direito a segundo pré-molar do lado esquerdo, após o tempo de ação foi removido com cânula de sucção, lavado abundantemente com água e removido a barreira gengival, todo esse procedimento foi realizado 3 vezes, com tempo entre cada sessão de 3 dias.

Figura 2: A) Top Dam - Barreira Gengival; B) Gel Clarendor Whiteness HP AutoMixx 35\%.
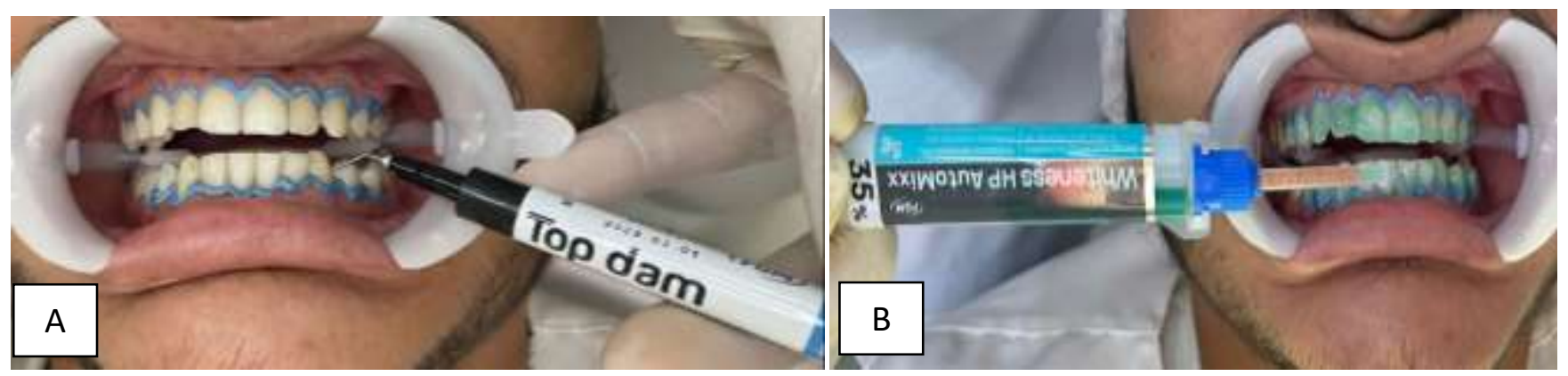

Fonte: Autores.

Após a primeira sessão o score de cor observado foi A2. Na segunda sessão foi realizando novamente a profilaxia com pedra pomes e água, registro de cor usando a escala de cor Vita Pan (Classical B1 - D4) com score A2 e após a segunda sessão foi observado o score de cor A1 e registro fotográfico. Após a terceira sessão o resultado final de cor foi B1. As restaurações dos elementos 12 e 22 serão trocadas após 15 dias o tratamento clareador. As figuras 3 mostram os resultados alcançados 3+6partir da segunda cada sessão: 
Figura 3: A) Antes da $2^{\circ}$ sessão; B) Depois da $2^{\circ}$ sessão; C) Antes da $3^{\circ}$ sessão; D) Depois da $3^{\circ}$ sessão; E) Resultado final.

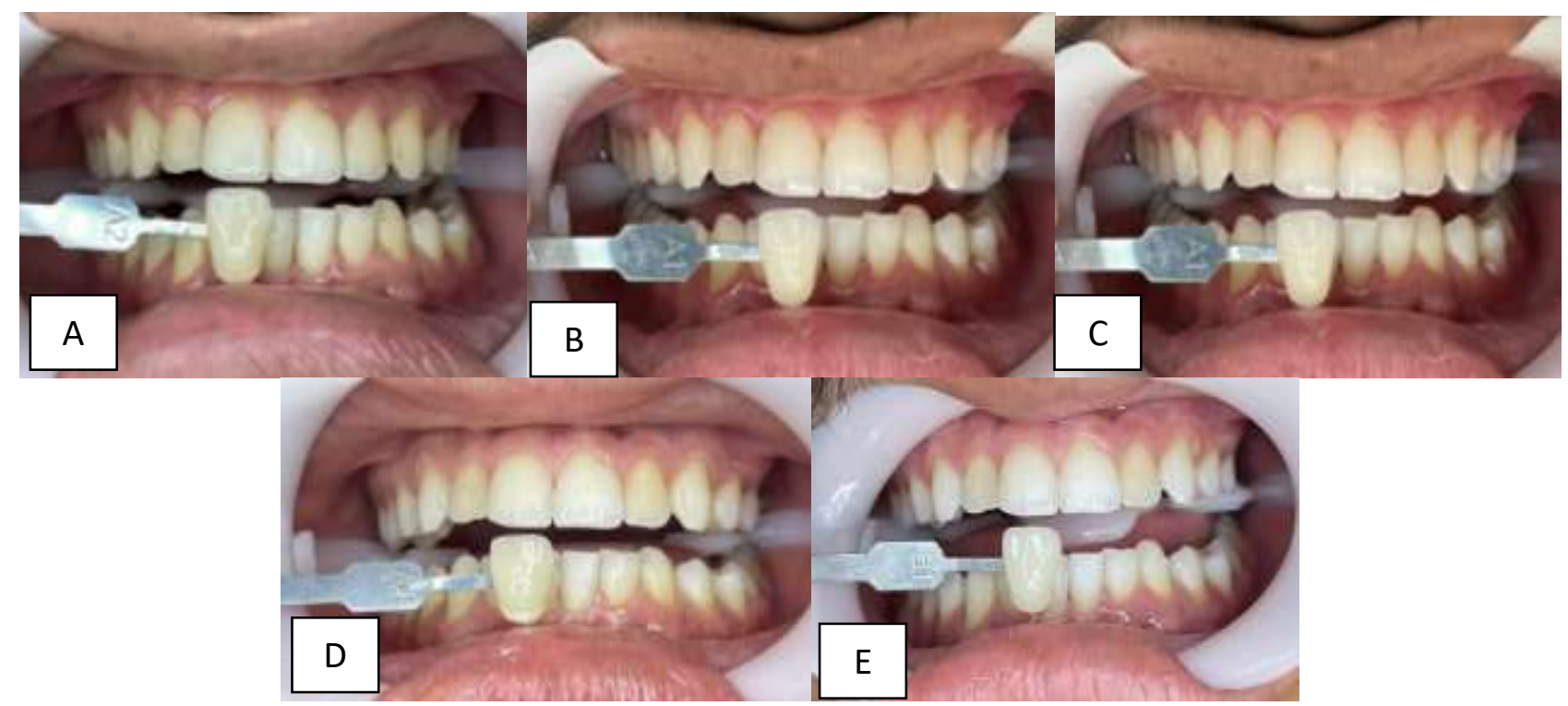

Fonte: Autores.

\section{Discussão}

As manchas em dentes podem ter vários fatores, tais como: medicamentos de uso intracanal, materiais restauradores, hemorragia intracoronária, decomposição de detritos, estes resíduos penetram nos túbulos dentinários provocando a descoloração dentária (Poluha et al., 2016). Existem várias condições fisiológicas que podem ser melhoradas esteticamente pelo clareamento dental. Dentre essas condições estão: as manchas de tetraciclina suaves, fluorose superficial, trauma, mortificação da polpa e pigmentações extrínsecas. Os agentes clareadores são divididos em duas categorias, aqueles utilizados no consultório dental pelo profissional e os caseiros, que são administrados pelo paciente com supervisão do profissional (Franci et al., 2015)

Barbosa et al., (2020) relatam que, as alterações podem ter relação com a penetração de sangue nos túbulos dentinários promovendo a pigmentação do elemento dentário. Vieira et al., (2018) o escurecimento tem como consequência hemorragia, por detritos que ficaram na câmara pulpar, ou seja, deterioração do tecido pulpar, ou até mesmo as restaurações dentárias podem ser a causa do escurecimento dentário. Já para Astolf et al., (2017) comentam que, o escurecimento dental pode ter relação com traumatismo, e a insatisfação da cor dentária, possibilita cada vez mais a procura por opções de clínica para ter um melhor padrão dentário de clareamento.

Neto et al., (2019) afirmam que, a alteração de cor dos dentes pode variar quando a sua gravidade e localização, assim sendo, a polpa é responsável pela manutenção da cor dentária bem como a perda de vitalidade podem afetar diversos graus de coloração e brilho dental tornando-se escurecido com diferentes tonalidades que vão de cinzas à esverdeados. Neste contexto Resende e Siqueira et al., (2020) comentam que, os dentes podem passar por alterações congênitas tais como a dentinogênese imperfeita, porfirizo congênito, hipoplasia dos esmaltes entre outras como a do esmalte, bem como o uso de medicamentos como tetraciclina e outras alterações de ordem sistêmica.

Deste modo Neto et al., (2019) afirmam que, são de extrema importância a seleção dos gentes clareadores, uma excelente anamenese, conhecimento etilógico dos pacientes assim como a analisar as particularides de cada paciente, tais como: os casos de pigmentação, idade, estado vital dos dentes a serem clareados. Já para Francci et al., (2010) comentam que, esta análise pode facilitar a seleção correta para tonalidade dos elementos dentais, contudo é imprescindível que o profissional tenha um olhar clínico bastante especifico, deixando os pacientes cientes dos resultados final do tratamento. 
Assim sendo Pohula et al., (2019), afirmam que, o clareamento dentário tem como principal objetivo viabilizar a harmonia estética e tem como vantagem a conservação da estrutura dos dentes, além disso é um procedimento que visa atender a expectativa do paciente. De acordo com Carlos et al., (2017) em seus estudos afirmam ainda que, existem trës técnicas: Consultório, Caseira e Mista. Já para Linhado et al., (2012) a técnica de consultório é realizada no consultório pelo cirurgião dentista, a técnica caseira, pelo próprio paciente e a mista é a associação de ambas as técnicas. Corroborrando Barbosa et al., (2015) adverte que no clareamento caseiro deve-se observar a confecção de uma moldeira para que o paciente aplique o gel clareador, conforme as instruções do profissional de odontologia. Já Francci et al., (2000) comentam que o clareamento de consultório, é feito na clínica sendo acompanhado em todo o tratamento por um cirurgião dentista, permitindo assim o acompanhamento e as intervenções necessárias.

Para Carlos et al., (2000) este tipo de tratamento é considerado uma técnica não invasiva que possibilita ao cirurgião dentista corresponder às expectativas dos pacientes em busca de dentes mais claros. Para Da Silva et. al., (2020) enfatizam que o processo de tratamento em consultório a concentração de peróxido de hidrogênio geralmente vária entre 25\% a 50\%. Já Kina et al., (2015) afirma que em relação ao hidróxido de carbamida é utilizado 35\% com ou sem fonte de luz acompanhado pelo cirurgião dentista.

Conforme Kina et al., (2015) o clareamento em consultório possibilita uma resposta rápida, pois o agente clareador é utilizado em maior concentração, em relação ao Peróxido de Hidrogênio é utilizado em 35\%, contudo mais indicado em pequenos grupos dentais. Geralmente em apenas algumas consultas e sessões adequadas atinge-se os resultados esperados.

Carlos et al., (2000) comentam que, a utilização do peróxido de hidrogênio 35\% (Whitness HP Automixx / FGM) proporciona resultados favoráveis, possibilitando ao paciente uma estética no sorriso satisfatório, com uma mudança significativa na coloração dentária.

No presente caso, foi utilizado o agente clareador de peróxido de hidrogênio a 35\% (Whitness HP Automixx / FGM) aplicado apenas uma vez em cada sessão por tempo de 50 minutos, entretanto o intervalo foi reduzido de sete dias para três dias, diminuindo assim o tempo de tratamento de 21 dias para 09 dias.

De acordo com Castro et al., (2015) em seus ensaios foram realizadas três sessões de clareamento com ativação feita pelo LED, com intervalo de três dias entre uma sessão para outra. Já Tavares et al., (2021) enfatiza que em seus estudos foi utilizado o peróxido de hidrogênio $35 \%$ associado também ao ozônio ou luz ultravioleta, em todos os seus protocolos foram feitos com um intervalo de 48 horas entre as 3 sessões do tratamento. Soares et al., (2014) afirmam em seus achados que os resultados encontrados em seus pacientes foram positivos, o clareamento foi realizado em três sessões e avaliados no intervalo de 72 horas após o clareamento. Castro et al., (2015) comentam ainda que, ao final do tratamento a paciente apresentou coloração B1 na escala VITA. Já Soares et al., (2014); Tavares et al., (2021) comentam que utilização do peróxido de hidrogênio, em concentrações de $17 \%$ a $35 \%$ por até 45 minutos de aplicação podem ter resultados excelentes assim como reduzir os intervalos entre as sessões conforme a necessidade do paciente. Por outro lado, Castro et al., (2015) relatam que em seus estudos foi reduzido o percentual de concentração do peróxido de hidrogênio para 17\% uma vez que agentes clareadores mais concentrados podem causar maior sensibilidade ao paciente. Corroborando Tavares et al., (2021) comenta que este fato se deve à geração de maior pressão no espaço intracoronária, causada pelas moléculas de oxigênio liberadas a partir do peróxido de hidrogênio.

Neste contexto Costa et al., (2021) em seus estudos relatam que a hipersensibilidade no clareamento de consultório e bem maior comparando com o clareamento caseiro, uma vez que o peróxido de hidrogênio pode atingir a polpa de forma mais rápida em virtude da sua grande concentração. Já Rezende e Siqueira et al., (2014) evidenciam que no clareamento dental o fator responsável pela sensibilidade dentária assim como a alteração de cor é o princípio ativo do peróxido de hidrogênio. 
Neste relato o paciente não apresentou sensibilidade durante ou após o clareamento dentário no decorrer de todas as sessões. Contudo de acordo com Tavares e al., (2020) o peróxido de hidrogênio a 35\% pode resultar um clareamento eficiente possibilitando diminuir o intervalo para avaliar os resultados de 7 dias para 3 dias entre as sessões. Já para Castro et al., (2015) esta redução no intervalo para retorno do paciente viabiliza um tratamento em menor tempo e a satisfação do paciente, que pode obter resultado em até em três sessões proporcionando um clareamento eficaz e reduzindo o intervalo de tempo entre as sessões, possibilitando assim um tratamento em menor tempo e com qualidade. Já Carlos et al., (2000) afirmam que é uma técnica eficiente que não promove danos aos tecidos moles e duros da cavidade bucal e proporciona um clareamento eficaz.

Desta forma Castro et al., (2015); Tavares et al., (2020); Soares et al., (2014) Da Silva et al., (2020) em seus estudos mencionam que a utilização do peróxido de hidrogênio a 35\% utilizado no clareamento de dentes vitais amarelados ou escurecidos geralmente é realizado o tratamento em 3 sessões, entretanto enfatizam ainda, que pode variar o intervalo de tempo entre cada sessão em 48 horas ou 72 horas, quando há ausência de sensibilidade, promovendo resultados satisfatórios em menor tempo de tratamento.

\section{Considerações Finais}

Neste relato de caso clínico observou que o clareamento dental pode ser realizado de forma eficaz com redução no intervalo de tempo entre as sessões para três dias, quando o paciente não relatar sensibilidade, possibilitando assim mais rapidez no processo de clareamento dentário em consultório.

\section{Referências}

Barbosa, D. C., De’Stefan, T. R., Ceretta, L. B., Ceretta, R. A., Simoes, P. W., \& Dáltoe, L. F. (2015). Estudo comparativo entre as técnicas de clareamento dental em consultório e clareamento dental caseiro supervisionado em dentes vitais: uma revisão de literatura. Rev. Odontol. Univ. Cid. São Paulo.

Barbosa,T. B., Tavares, R. F., Batista, N. K., Lima, C. T., Cabral, L. L., \& Rezende, A. T. (2020). É necessário a restrição de corantes durante o clareamento? Ciências Biológicas e de Saúde Unit, Alagoas, 6(1), 21-32.

Basting, R. T., Amaral, F. L. B., França, F. M. G., \& Florio, F. M. (2012). Clinical Comparative Study of the Effectiveness of and Tooth Sensitivity to 10\% and $20 \%$ Carbamide Peroxide Home-use and 35\% and 38\% Hydrogen Peroxide In-office Bleaching Materials Containing Desensitizing Agents. Oper Dent, 37 (5), 464-473.

Carlos, N. R., Bridi, E. C., Amaral, F. L. B., Franca, F. M. G., Turssi, C. P., \& Alinhado, R. T. (2017). Eficácia do branqueamento doméstico Agentes entregues em formato personalizado ou Bandejas descartáveis pré-preenchidas: A Ensaio clínico randomizado. @ Odontologia Operatória, 42-1, 30-40

Castro, S. S., Leal, C. L., Argolo, S., Azedo, J. F., Mathias, P., \& Cavalcanti, A. N. (2015). Clareamento dental em pacientes com hipersensibilidade. Revista Bahiana de Odontologia, 6(1), 58-69.

Caviedes-Bucheli, J., Gernan, A-G., Mendez, S. R., Osorio, N. R., Lombana, N., \& Munhoz, H. R. (2008). The Effect of Tooth Bleaching on Substance P Expression in Human Dental Pulp. Journal of Endodontics. 34, Issue 12, Pages 1462-1465.

Costa, E. I. D., Lopes, T. Q., Cruz, J. H. A., Guenes, G. M. T., Medeiros, L. A. D. M., Penha, E. S., Almeida, M. S. C., \& Figueiredo, C, H. M. C. (2021). Clareamento dental de consultório e sensibilidade: relato de caso. Arch Health Invest, 10(1), 72-77.

Da Silva, A. M. D., Nobre, F. C., Silva, M. M., Paulino, B. A. V., Ramos, A. T. P. R., \& Cabral, L. L. (2020). Clareamento caseiro ou clareamento de consultório foto iluminado: qual a técnica mais efetiva? Ciências Biológicas e de Saúde Unit, Alagoas, 6(1), 33-46

De Souza, T. S., De Queiroz, A. M., Araujo, M. R.A., Verissimo, M. H. G., Marques, S. C. S., Leal, T. R. B., \& Leal, T. N. R B. (2020). Métodos de clareamento dental para o sucesso clínico: uma análise comparativa de estudos clínicos através de uma revisão integrativa da literatura. Research, Society and Development, $10(3)$.

Franci, C., Marson, F. C., Briso, A. L. F., \& Gomes, M. N. (2010). Clareamento dental - Técnicas e conceitos atuais. Rev. Assoc. Paul. Cir. Dent, Ed esp(1), $78-8$

Kielbassa, A., Maier, M., Gieren, A-K., \& Eliav, E. (2015). Tooth sensitivity during and after vital tooth bleaching: A systematic review on an unsolved problem. Quintessence International, 46.

Kina, M., Borgh, A. P. S., Fabre, A. F., Martins, O. C. L., Simonato, L. E., Boer, N. P., \& Kina, J. (2015). Clareamento dental em dentes vitais: protocolo clínico em consultório. Arch Health Invest, 4(4), 7-12.

Lima, L. F., Alencar, A. H. G., Decurcio, D. A., Silva, J, A., Favarao, I. N., Loureiro, M. A. Z., Barletta, F. B., \& Estrela, C. (2019). Efeito do clareamento dental na polpa saturação de oxigênio na maxila central incisivos - um ensaio clínico randomizado. J Appl Oral Sci. 
Research, Society and Development, v. 10, n. 15, e594101523833 2021

(CC BY 4.0) | ISSN 2525-3409 | DOI: http://dx.doi.org/10.33448/rsd-v10i15.23833

Linhado, R., TAmaral. F. L. B., Franca. F. M. G., \& Florio. F. M. (2012). Estudo Clínico Comparativo da eficácia de e Sensibilidade do dente a $10 \%$ e $20 \%$ de peróxido de carbamida Uso doméstico e 35\% e 38\% Peróxido de hidrogênio Branqueamento em consultório Materiais contendo Agentes dessensibilizantes. Operative Dentistry, 37-5, 464-473.

Markowitz, V. M., Palaniappan, K., Chu, K., Szeto, E., Grechkin, Y., Ratner, A., Anderson, I., Lykdis, A., \& Mavromatis, K. (2010). Integrated microbial genomes system: an expanding comparative analysis resource. Nucleic Acids Research, 38, Issue suppl1, D382-D390,

Nascimento, J., \& Aracuri, T. (2018). Avaliação da eficácia entre os métodos de clareamento dental caseiro x de consultório - Revisão de Literatura. FACIPLAC, Gama-DF.

Pereira, A. S., Shitsuka, D. M., Parreira, F. J., \& Shitsuka, R. (2018). Metodologia da pesquisa científica. UFSM.

Poluha, R.L., Neto, C.L.M.M., Souza, B.M., Fialho, L.M., Yoshida, N.Y., \& Abio, S.S. (2016). Clareamento dental como aprimoramento estético do sorriso. 48, 94-98. Revista UNINGÁ, <http://www.scielo.aprimoramento_estetitca.sorriso.pdf>

Rezende, M., \& Siqueira, S. H. (2014). Clareamento dental - efeito da técnica sobre a sensibilidade dental e efetividade. Revista Assoc. Paulista de Cir. Dentis. 68(3), 208-12.

Santana. G. O., \& Silva. M. J. A. (2019). Clareamento Dental e a Influência da Dieta na Estabilidade da Cor: Revisão de Literatura. Id on Line Rev. Mult. Psic. 13(48), 268-279.

Silva Neto, J. M., De Aquino, J. M., Da Silva, B. R., Barros, K. F., Medeiros, M. L. B. B., \& Barros. J. V. B. A. R. (2020). Clareamento dental, aplicação em dentes vitais: uma revisão de literatura. Revista Eletrônica Acervo Saúde. REAS/EJCH, Sup. 47.

Soares, D. G., Basso, F. G., Hebling, J., \& Costa, C. A S. (2014). Concentrações e protocolos de aplicação para géis de branqueamento de peróxido de hidrogênio: efeitos na polpa viabilidade celular e eficácia de clareamento. Journal of dentistry, 42 - 185-198.

Tavares, N. R. N. O., Galvao, A. Da Mata., Dietric, L., Carvalho, R. F., Silva, R. S., Silva, A. L. F., \& Da Silva, G. R. (2021). O ozônio ou a luz violeta podem melhorar a mudança de cor ou físico-química propriedades do peróxido de hidrogênio dente branqueado? Revista Brasileira de Odontologia, 32(4). $19-30$.

Vieira. J. G., Reis. J. A. B., Mondelli. R. F. L., \& Soares. A. F. (2019). Efeitos do clareamento dental em consultório para dentes polpados: uma revisão da literatura. SALUSVITA, Bauru, 38(3), 739-754. 